\title{
Variation in Cuticular Hydrocarbons among Strains of the Anopheles gambiae sensu stricto by Analysis of Cuticular Hydrocarbons Using Gas Liquid Chromatography of Larvae
}

\author{
Greg I Anyanwu ${ }^{+}$, David H Molyneux*, Angela Phillips** \\ Department of Biological Sciences, University of Salford, England, UK *School of Tropical Medicine, Pem- \\ broke Place, Liverpool L3 5QA, England, UK **School of Sciences, Staffordshire University, College Road, \\ Stoke-on-Trent ST4 2DE, England, UK
}

Cuticular hydrocarbons of larvae of individual strains of the Anopheles gambiae sensu stricto were investigated using gas liquid chromatography. Biomedical discriminant analysis involving multivariate statistics suggests that there was clear hydrocarbon difference between the Gambian(G3), the Nigerian (16CSS and, its malathion resistant substrain, REFMA) and the Tanzanian (KWA) strains. The high degree of segregation (95\%) in hydrocarbons among the four strains investigated indicates that further analysis is needed to enable understanding of hydrocarbon variation in samples of An. gambiae especially from areas where these populations co-exist.

Key words: Anopheles gambiae sensu stricto strains - cuticular hydrocarbon analysis

The morphological similarity of the members of the Anopheles gambiae complex has posed a problem to identification. Species A, B, and C of the complex were originally identified by their mating incompatibility by cross-mating with known species (Davidson 1964). Since then other techniques have been applied in the differentiation of the cryptic species including (a) detection of differential banding patterns of the polytene chromosomes from the ovarian nurse cells of the semi-gravid adult females (Coluzzi \& Sabatini 1967); (b) examination of the fluorescent banding sequences of mitotic sex chromosomes (Gatti et al. 1977); (c) evaluation of electrophoretic isoenzyme frequencies (Mahon et al. 1976, Miles 1978); (d) analysis of differences in cuticular hydrocarbons by chromatography (Carlson \& Service 1979, 1980, Hamilton \& Service 1983); and (e) using DNA characteristics (dot hybridisation technique) for distinguishing adults of An. gambiae sensu stricto (s.s.) (Gale \& Crampton 1987) as well as (g) using ribosomal RNA gene probe to differentiate members of the An. gambiae complex (Collins et al. 1987).

\footnotetext{
This research received financial support from the UNDP/ World Bank/WHO Special Programme for Research and Training in Tropical Diseases.

${ }^{+}$Corresponding author. Current address: Applied Entomology and Parasitology Unit, Department of Zoology, University of Jos, P.M.B. 2084, Jos, Nigeria.

E-mail: anyanwug@unijos.edu.ng

Received 17 May 1999

Accepted 6 December 1999
}

Differentiation of larvae and adult females of both salt and mineral water species of most individuals of the complex have made use of anatomical features of pecten, setal branching, antennal coeloconica, palpal ratios, tarsal, palpal and wing markings as well as thoracic scales (White 1974). Apart from distinct egg morphology (Ramsdale \& Leport 1967), all these morphological and meristic features showed much variation both in dimension and ratios. For the freshwater species, the only reliable means of identification until recently was almost entirely on chromosome analysis. This study is the first to use hydrocarbon analysis to discriminate larval mosquitoes.

The present investigation was prompted by the need to provide further information on the differentiation of members of the gambiae complex and the need to ascertain if the cuticular hydrocarbon technique which had been successfully applied for the identification of An. gambiae s.s. (Carlson \& Service 1980), chromosomal forms of An. gambiae s.s. and An. arabiensis (Phillips et al. 1987), adults of An. culicifacies (Milligan et al. 1986) and An. maculipennis (Phillips et al. 1990), strains of An. stephensi (Anyanwu et al. 1993), would be appropriate for separating the strains of An. gambiae s.s.

\section{MATERIALS AND METHODS}

Experimental mosquitoes - Samples of larvae were taken from adult colonies established from collections of An. gambiae s.s. at the London School of Tropical Medicine. They consist of four strains viz G3- originating from The Gambia, West Africa and colonized in London since 1975; 16CSS, derived in 1974 from wild caught adults 
originating from Lagos, Nigeria (West Africa); KWA, strain from Kwale, Tanzania (East Africa) colonized in London since 1975; REFMA, colony formed in London since 1979 thought to be a substrain of 16CSS resistant to malathion.

Solvent extraction of cuticular lipid - Cuticular lipid extraction essentially followed the technique of Phillips et al. (1988) which involved dipping each dead dry/desicated mosquito larva in $10 \mu \mathrm{ln}$ hexane (spectrophotometric grade) at room temperature for $1 \mathrm{~min}$ to give enough cuticular wax for hydrocarbon analysis without undue contamination by the insect's internal lipids; evaporating the extract to dryness, adding $2 \mu \mathrm{l}$ of $10 \mathrm{ppm}$ pentadecane $\left(\mathrm{C}_{15} \mathrm{H}_{32}\right)$ to the concentrated sample extract as a reference standard to aid quantification of the hydrocarbon components in each extract as well as ensure reproducibility from sample to sample and, after $1 \mathrm{~min}$ of resuspension, injecting the entire extract unto the capillary column.

Chromatography - Chromatographic analysis used a Perkin Elmer 8420 machine fitted with a flame ionisation detector (FID). Oven temperatures were programmed to start at $75^{\circ} \mathrm{C}$, rise at a ramp rate of $25^{\circ} \mathrm{C} / \mathrm{min}$ to $120^{\circ} \mathrm{C}$ for $2 \mathrm{~min}$ and then rise again at $15^{\circ} \mathrm{C} / \mathrm{min}$ to $310^{\circ} \mathrm{C}$ for $15 \mathrm{~min}$. Helium, flowing at $6.8 \mathrm{ml} / \mathrm{min}$ through the column, was used to convey the hydrocarbon constituents to the Flame Ionisation Detector where the deflections they cause is recorded in the integrator and are reflected as peaks in the resulting chromatogram. GLC analysis was initiated when each resuspended extract was loaded directly onto the column injector using a microsyringe.

Discriminant analysis - The hydrocarbon peaks emerging from each sample extract were numbered by comparison to known hydrocarbon standards for case of recognition. The area under each peak on the chromatogram represented the concentration of a hydrocarbon component in the extract. The concentrations of each component were calculated by comparing the area under each peak with that of the internal standard, and these formed the data for statistical analysis.

Multivariate analysis of variance using the BMDP 7M package of Dixon (1988) was then applied to test for significant differences in the hydrocarbon quantity between the samples (Phillips et al. 1988, Anyanwu et al. 1993). To aid this hydrocarbon characterisation, the F-to-enter ratio was set at 6.0, a conservative value, which best enhances differences between groups in addition to reducing to reasonable level the number of variables needed to separate groups. This discriminant package further employs a jack-knifed ("leave-one-outand then classify") estimator to gauge the proportion of correct classifications made by the discrimi- nant analysis. Here each individual involved in the classification is left out each time the classification function used to assign it to a group is being computed. This serves as an empirical measure of the assessment of successful classification.

\section{RESULTS}

Overall, 185 mosquito larvae were analyzed (52 each of 3 strains, G3, 16CSS and KWA and 29 of the REFMA strain) each giving a GC profile (Fig. 1) for further analysis. Discriminant analysis indicated that, of a profile of 80-100 peaks, variability in hydrocarbon composition between individuals of An. gambiae s.s. strains is dependent on peaks $7,17,22,33,34,35,44,45$ and 47 . Visually systematic differences between individual peaks may be difficult to estimate. Nonetheless, it can be seen, for instance, that peak 7 is more pronounced in KWA and REFMA but reduced in G3 and 16CSS. Again, whereas peaks 17 and 45 are more prominent in KWA than in the others, peak 22 is smaller in REFMA than in the rest. Peak 47 is conspicuous in 16CSS and REFMA, peak 44 is diminished in all the cases.

For segregation among the four strains, three classification functions have been created from the data analysis (Table I). How well these groups separate from one another depends on the impact made by the different variables on the discriminant functions. Of the peaks selected in this analysis, four of them: 22, 17, 47 and 33 in that order, showed the highest loading on the first discriminant function (Table I). These four peaks account for the separation of G3 and KWA strains. This impact is reflected on their rating on the canonical variables evaluated at group means (Table II). On the first discriminant function KWA group is some distance from the rest, while 16CSS and REFMA are nearly inseparable.

On the other hand, the second discriminant function is affected largely by values on peaks 47 and 33. On this function, REFMA is separated from the other groups. Similarly the highest contribution to the third discriminant function was made by peaks 44,5 and 17 . This function is responsible for the separation of G3 and 16CSS. Thus on the 1 st d.f, the KWA group is some distance from the rest but 16CSS and REFMA are close together (Table II). On the second discriminant function REFMA is far removed from G3 and 16CSS. It takes both discriminant functions to separate KWA, REFMA from G3 and 16CSS.

By plotting individuals according to their scores on the first two discriminant functions which act as rectangular coordinates, a scatter plot of the type shown in Fig. 2 gives a graphic illustration of the spacial disposition of the four groups relative to one another and hence a picture of the extent of 
Anopheles gambiae Strains
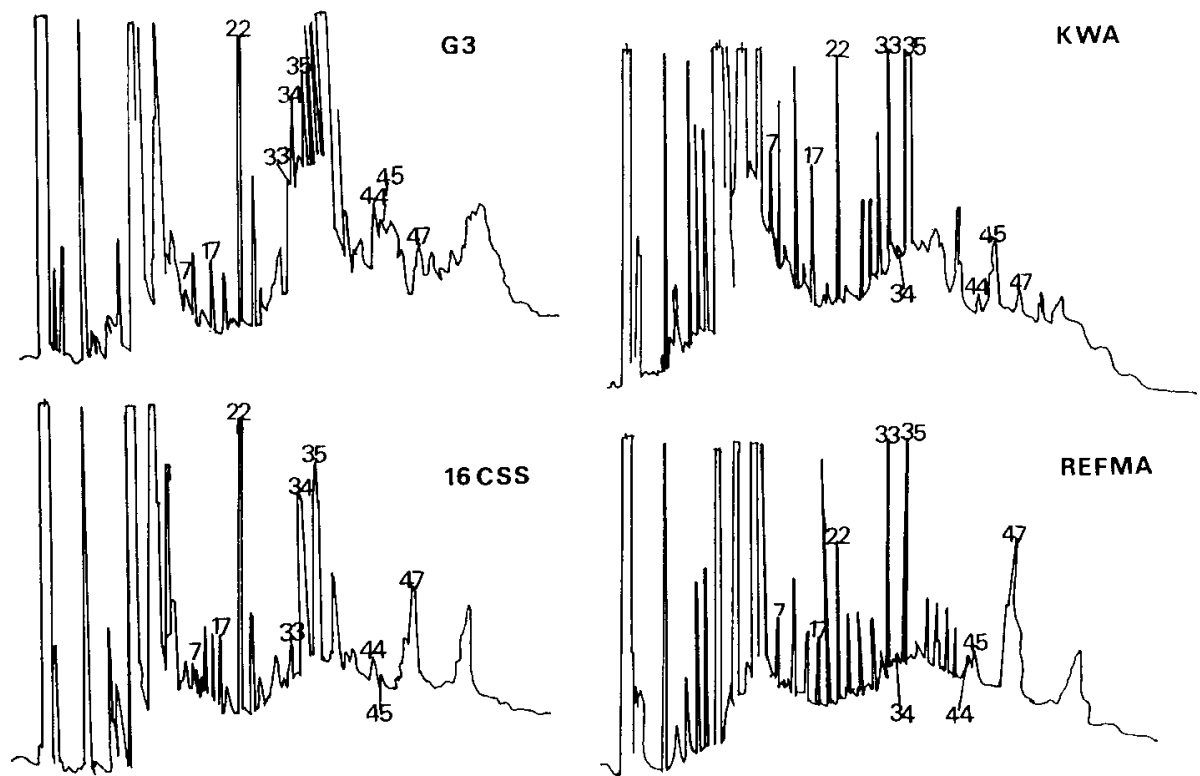

Fig. 1: gas chromatography profile of four strains of Anopheles gambiae sensu stricto hydrocarbons, showing the subset of peaks which accounted for the segregation larvae.

TABLE I

Discriminant function coefficients for distinguising the four strains of Anopheles gambiae

Standardised (by pooled within variance) coefficients for canonical variables

\begin{tabular}{cccc} 
Component & & & \\
\cline { 2 - 4 } number & 1 & 2 & 3 \\
\hline 5 & -0.07652 & 0.01292 & -0.58149 \\
17 & -0.54594 & 0.36139 & 0.39629 \\
22 & 0.70051 & 0.09396 & -0.08855 \\
33 & -0.17367 & -0.64507 & 0.28374 \\
34 & 0.15892 & -0.03698 & -0.033580 \\
44 & 0.14880 & 0.23765 & 1.34963 \\
45 & -0.14880 & -0.007 & -0.32718 \\
47 & 0.21383 & -0.00907 & 0.34302 \\
\hline
\end{tabular}

TABLE II

Canonical variables (evaluated at group means) for the four strains of Anopheles gambiae

\begin{tabular}{lrrr}
\hline & \multicolumn{3}{c}{ Discriminant function } \\
\cline { 2 - 4 } Group & \multicolumn{1}{c}{1} & \multicolumn{1}{c}{2} & \multicolumn{1}{c}{3} \\
\hline G3 & 1.68340 & 0.90735 & 1.20754 \\
16CSS & 0.88845 & 0.67331 & -1.49503 \\
KWA & -3.03764 & 0.20797 & 0.24391 \\
REFMA & 0.83521 & -3.20720 & 0.07814 \\
\hline
\end{tabular}

hydrocarbon discrimination between the strains investigated.

Jack-knifed estimators of the proportion of correct identifications was used to test the accuracy of the classifications made by the discriminant analysis; 95\% correct segregation among the four strains of An. gambiae s.s. larvae was achieved (Table IV)

\section{DISCUSSION}

Using discriminant analysis contrasts in hydrocarbons have been demonstrated among the larvae of four strains of An. gambiae s.s. from the 95\% success rate in their discrimination. The results indicate, for example, that the hydrocarbons of the Gambian (G3), Nigerian (16CSS and its malathion resistant sub-strain, REFMA) and the Tanzanian (KWA) strains of An. gambiae s.s. are different.

The low levels of misclassification of populations of An. gambiae s.s. indicates that some individuals from different strains share identical hydrocarbons or this may be due to the correlated nature of the variables responsible for their separation or a reflection of interrelatedness of the strains (notice overlap in distribution between G3 and 16CSS strains: Fig. 2). Lockey (1976) had demonstrated that marked similarities in hydrocarbon profiles was common even among distantly related species. 


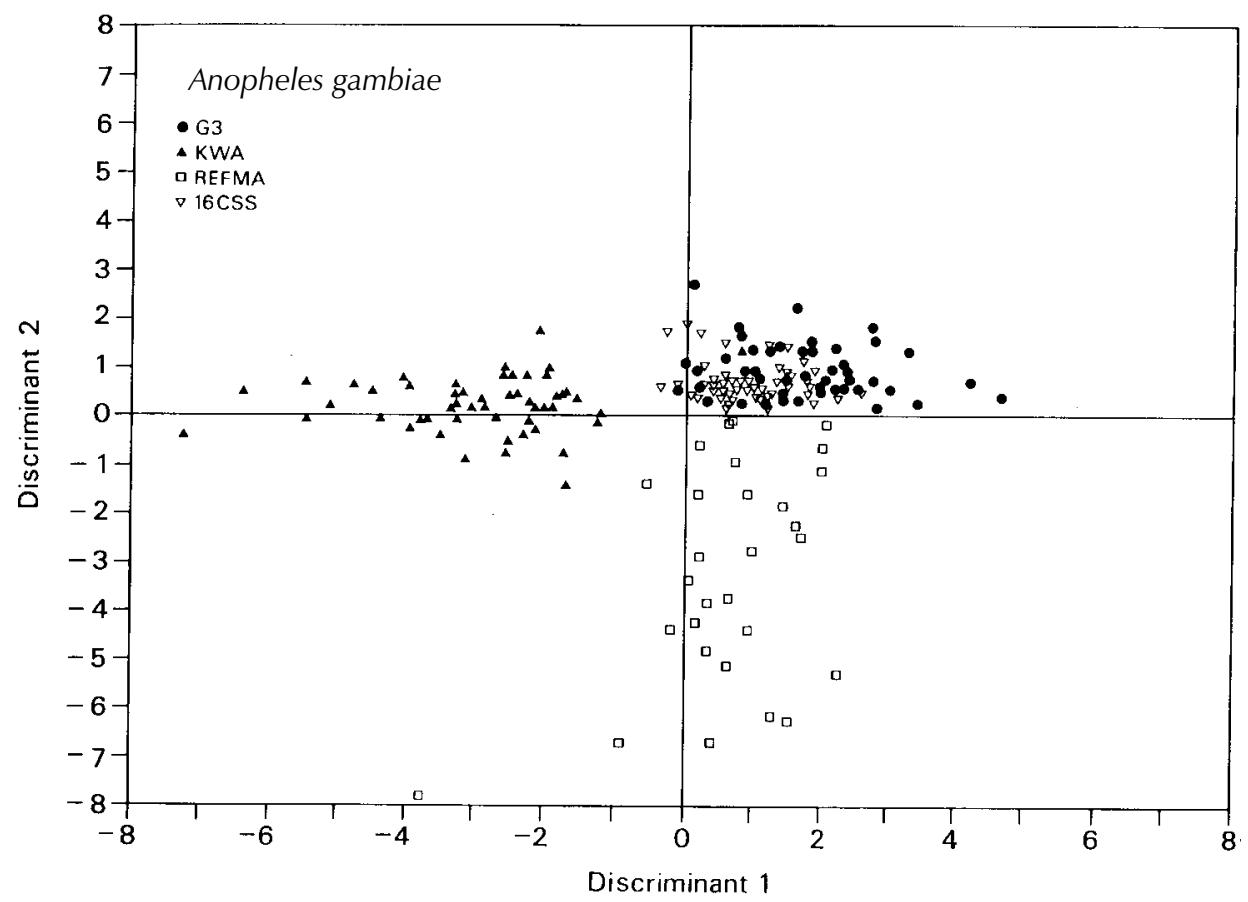

Fig. 2: discriminant function plot showing the distribution of individuals of four Anopheles gambiae s.s. strains.

Hydrocarbon differences amongst strains of the gambiae species analyzed here could be considered as reflecting geographical variation. For instance, G3 strain (from The Gambia) could be distinguished from 16CSS (from Nigeria) with a 97\% confidence rate. There was $100 \%$ segregation between the Nigerian (16CSS) and the Tanzanian (KWA) strains. From pairwise comparisons (Table IV), it has been possible to separate any two strains within the An. gambiae species with an average success rate of $95 \%$.

The matrix of distance (Mahalanobis') between the groups lends further support to the hydrocarbon relationship among the strains of An. gambiae s.s. studied (Table V). G3 strain appears to show greater resemblance to the Nigerian strain (16CSS), being separated from it by 22.06 whereas its distance from KWA is as much as 65.46.

Identification of cryptic species has capitalised on intraspecific differences in the hydrocarbons of the insect cuticle. This has been found useful in particular for the differentiation of sibling species of Anopheles mosquitoes (Carlson \& Service 1979, 1980, Hamilton \& Service 1983, Milligan et al. 1986), Simulium blackflies (Carlson \& Walsh 1981) and in the sandflies (Ryan et al. 1986).

Although the biological implications of variations in cuticular hydrocarbons between very closely related species have not been fully stud- ied. Phillips and Milligan (1986), Phillips et al. (1987) and Milligan et al. (unpubl. data) suggest that within such relationships species-specific differences do exist which tend to highlight the effect of geographical variation and possible incipient speciation mechanisms.

The species-specific function performed by insect cuticular hydrocarbons in various environments have been outlined by Howard and Blomquist (1982) and Phillips et al. (1988). They serve mainly to prevent dessication and to assist in "chemical communication" (Lockey 1980, Howard \& Blomquist 1982).

Discrimination between the strains of $A n$. gambiae, here analysed, was based on the quantities of various hydrocarbons (Table III). Milligan et al. (1990 unpubl. data) argued that the hydrocarbon discrimination is based on the relative concentrations of the component chemicals, rather than their mere presence or absence. Similarly, Hamilton and Service (1983) observed that, although the fourth instars of An. gambiae and An. arabiensis had similar cuticular hydrocarbons, there was still differences in the relative levels of some of these chemicals.

It is thought that hydrocarbons are not just passive protectors against desication, they may additionally play a leading role in mate selection/recognition and population divergence. Hydrocarbon 
TABLE III

Jack-knifed classification scores showing the number of individuals of Anopheles gambiae correctly allocated to their strain

\begin{tabular}{lcllll}
\hline & & \multicolumn{4}{c}{ No. of cases classified into } \\
\cline { 3 - 6 } Group & No. & G3 & 16 CSS & KWA & REFMA \\
\hline G3 & 52 & 49 & 3 & 0 & 0 \\
& & $(94.23 \%)$ & $(5.77 \%)$ & $(0.0 \%)$ & $(0.05)$ \\
16CSS & 52 & 0 & 52 & 0 & 0 \\
& & $(0.0 \%)$ & $(100.0 \%)$ & $(0.0 \%)$ & $(0.0 \%)$ \\
KWA & 52 & 0 & 0 & 52 & 0 \\
& & $(0.0 \%)$ & $(0.0 \%)$ & $(100.0 \%)$ & $(0.0 \%)$ \\
REFMA & 29 & 2 & 4 & 0 & 23 \\
& & $(6.90 \%)$ & $(13.7 \%)$ & $(0.0 \%)$ & $(79.31 \%)$ \\
\hline Total & 185 & & & & \\
\hline
\end{tabular}

$\%$ correct $($ classification $)=95.1 \%$

\section{TABLE IV}

Result of pairwise discrimination among strains of Anopheles gambiae based on their hydrocarbon characters

\begin{tabular}{llll}
\hline & G3(A) & $16 \mathrm{CSS}(\mathrm{B})$ & $\mathrm{KWA}(\mathrm{C})$ \\
\cline { 2 - 3 } $16 \mathrm{CSS}(\mathrm{B})$ & 97.1 & & \\
KWA (C) & 99.0 & 100 & \\
REFMA(D) & 95.1 & 97.5 & 98.8 \\
\hline
\end{tabular}

\section{TABLE V}

F-matrix indicating the Mahalanobis' measure of distance between the strains of Anopheles gambiae studied

\begin{tabular}{llll}
\hline & G3 & 16CSS & KWA \\
\hline 16CSS & 22.06 & & \\
KWA & 65.46 & 51.51 & \\
REFMA & 37.42 & 34.67 & 32.77 \\
\hline
\end{tabular}

differences among sympatric populations of $A n$. gambiae s.s. (Phillips et al. 1987, Milligan et al. 1990 unpubl.) might reflect a semiological function of the compounds enabling the insect to recognise potential mates in those locations where sibling species coexist. In some insects, part of the mate recognition mechanism has been linked with the detection of specific hydrocarbon compounds and other components of the cuticular lipid layer e.g fatty acids, alcohols, sterols, aldehydes etc. (Jallon 1984, Bonavita-Courgourdan et al. 1987, Pescke 1987).

Although our limited sample analysis here using laboratory colonies of An. gambiae s.s. provides evidence for intraspecific hydrocarbon differences, this variation could have wider implica- tions for populations in the wild. It is not certain the extent to which hydrocarbons may be influenced by genetic, ecological and other geographical differences particularly in the wild. However further analysis of samples of An. gambiae drawn from different populations especially in areas where members of the sibling species coexist, will help provide a clearer picture.

\section{ACKNOWLEDGEMENTS}

To the London School for providing the mosquito specimens for this analysis; to Dr Ron Lawson, Chairman, Department of Biological Sciences, Salford University, for allowing us the use of facilities, and Dr Mike Wilson, for help with the statistical analysis.

\section{REFERENCES}

Anyanwu GI, Davies DH, Molyneux DH, Phillips A, Milligan PJ 1993. Cuticular hydrocarbon discrimination/variation among strains of the mosquito, Anopheles (Cellia) stephensi Liston. Ann Trop Med Parasitol 87: 269-275.

Bonavita-Courgourdan A, Clement JL, Lange C 1987. Nestmate recognition: the role of cuticular hydrocarbons in the ant, Camponotus vagus Scop. J Entomol Sci 22: 1-10.

Carlson DA, Service MW 1979. Differentiation between species of the Anopheles gambiae complex (Diptera: Culicidae) by analysis of cuticular hydrocarbons. Ann Trop Med Parasitol 3: 589-592.

Carlson DA, Service MW 1980. Identification of mosquitoes of Anopheles gambiae species complex a and b by analysis of cuticular components. Science 207 : 1089-1091.

Carlson DA, Walsh JP 1981. Identification of two West African blackflies (Diptera: Simuliidae) of the Simulium damnosum species complex by analysis of cuticular paraffins. Acta Trop 38: 235-239.

Collins FH, Mendez MA, Razmussen MO, Mehaffey PC, Besansky NJ, Finnerty V 1987. A ribosomal RNA gene probe differentiates member species of 
the Anopheles gambiae complex. Am J Trop Med Hyg 37: 37-41.

Coluzzi M, Sabatini A 1967. Cytogenetic observation on the species a and $\mathrm{b}$ of the Anopheles gambiae complex. Parassitologia 9: 73-88.

Davidson G 1964. The five-mating types in the Anopheles gambiae complex. Riv Malariol 43: 167-183.

Dixon WJ 1988. BMDP Statistical Software Manual (1988 Software Release), vol 1, University of California, Berkeley.

Gale KR, Crampton JM 1987. DNA probes for species identification of mosquitoes in the Anopheles gambiae complex. Med Vet Entomol 1: 127-136.

Gatti M, Santini G, Pimpinelli S, Coluzzi M 1977. Fluorescence banding techniques in the identification of sibling species of the Anopheles gambiae complex. Heredity 38: 105-108.

Hamilton RJ, Service MW 1983. Value of cuticular and internal hydrocarbons for the identification of larvae of Anopheles gambiae Giles, Anopheles arabiensis Patton, and Anopheles melas Theobald. Ann Trop Med Parasitol 77: 203-210.

Howard RM, Blomquist GJ 1982. Chemical ecology and biochemistry of insect hydrocarbons. Annu Rev Entomol 27: 149-172.

Jallon JM 1984. A few chemical words exchanged by Drosophila during courtship and mating. Behav Genet 14: 441-478.

Lockey KH 1976. Cuticular hydrocarbons of Locusta, Schistocerca and Periplaneta and their role in waterproofing. Insect Biochem 6: 457-472.

Lockey KH 1980. Insect cuticular hydrocarbons. Comp Biochem Physiol 65B: 457-462.

Mahon RJ, Green CA, Hunt RH 1976. Diagnostic allozymes for routine identification of adults of the Anopheles gambiae complex (Diptera: Culicidae). Bull Entomol Res 68: 85-96.

Miles SJ 1978. Enzyme variation in the Anopheles gambiae group of species (Diptera: Culicidae). Bull Entomol Res 68: 85-96.
Milligan PJM, Phillips A, Molyneux DH, Subbarao SK, White GB 1986. Differentiation of Anopheles culicifacies Giles (Diptera: Culicidae) sibling species by analysis of cuticular components. Bull Entomol Res 76: 529-537.

Pescke K 1987. Cuticular hydrocarbons regulate mate recognition, male aggression and female choice of the rove beetle, Aleochara curtula. J Chem Ecol 13: 993-2008.

Phillips A, Milligan P 1986. Cuticular hydrocarbons distinguish sibling species of vectors. Parasitol Today 2: $180-181$.

Phillips A, Milligan PJM, Broomfield G, Molyneux DH 1988. Identification of medically important Diptera by analysis of cuticular hydrocarbons. In MW Service, Biosystematics of Haematophagous Insects, Clarendon Press, Oxford, p. 39-59.

Phillips A, Milligan PJM, Coluzzi M, Toure Y, Broomfield G, Molyneux DH 1987. Studies of the chromosomal forms of Anopheles gambiae s.str. and Anopheles arabiensis using cuticular hydrocarbons. In Proceedings of the 3rd International Conference on Malaria and Babesiosis, Annecy, France, p.164.

Phillips A, Sabatini A, Milligan PJM, Boccolini D, Broomfield G, Molyneux DH 1990. The Anopheles maculipennis complex (Diptera: Culicidae): comparison of the cuticular hydrocarbon profiles determined in adults of five palaeartic species. Bull Entomol Res 80: 459-464.

Ramsdale CD, Leport GH 1967. Studies of Anopheles gambiae complex in West Africa. Bull WHO 36: 494500.

Ryan L, Phillips A, Milligan P, Lainson R, Molyneux DH, Shaw JJ 1986. Separation of female Psychodopygus wellcomei and P. complexus (Diptera: Psychodidae) by cuticular hydrocarbon analysis. Acta Trop 43: 85-89.

White GB 1974. Anopheles gambiae complex and disease transmission in Africa. Trans R Soc Trop Med Hyg 68: 278-301. 